ИЗВЕСТИЯ АКАДЕМИИ НАУК ЭСТОНСКОИ ССР. ФИЗИКА МАТЕМАТИКА PROCEEDINGS OF THE ACADEMY OF SCIENCES OF THE ESTONIAN SSR. PHYSICS * MATHEMATICS

1987, 36, 2

удК 539.172 .3

Э. РЕАЛО, М. ХААС, В. ХИЖНЯКОВ

\title{
КОГЕРЕНТНОЕ ДВИЖЕНИЕ ЯДЕРНОГО ВОЗБУЖДЕНИЯ В МЁССБАУЭРОВСКОМ ПОГЛОТИТЕЛЕ
}

В настоящее время в оптической и гамма-резонансной спектроскопии значительно возрос интерес к временным аспектам переходных процессов, возникающих при прохождении излучений в резонансной среде (см., напр., $\left.\left[{ }^{1}\right]\right)$.

Основополагающей в этой области исследований является работа $\left[{ }^{2}\right]$, где в качестве начала отсчета времени служил момент регистрации $\gamma$-кванта энергии 122,4 кэВ, испущенного при заселении мессб́ауэровского уровня 14,4 кэB ядра ${ }^{5 /} \mathrm{Fe}$. Чднако основное внимание в $\left[^{2}\right]$ и в последующих экспериментальных и теоретических исследованиях было уделено случаю тонких резонансных поглотителей, где переходные процессы развертываются относительно быстро.

В нашей работе показано, что в поглотителе значительной толщины имеются особенноети в пропускании $\gamma$-излучения, связанные с движением ядерной поляризации (ядерный поляритон) по образцу.

Хорошо известно $[2,3]$, что временные зависимости интенсивности мёссбауэровского излучения можно описать в рамках простой классической модели. Согласно этой модели в точке $z$ поле первичного $\gamma$-излучения, испущенного ядром, расположенным в точке $-l(z+l \geqslant 0)$ и перешедшим в момент $t=0$ на мёссбауэровский уровень, совпадает с полем соответствующего классического осциллятора

$$
\begin{aligned}
E_{i}(t, z)= & E_{0} e^{-\gamma t / 2} \exp \left[i \omega_{0}\left(t-\frac{z+l}{c_{0}}\right)\right] \theta\left(t-\frac{z+l}{c_{0}}\right)= \\
& =\frac{E_{0}}{2 \pi i} \int_{-\infty}^{\infty} \frac{\exp \left[i \omega\left(t-\frac{z+l}{c_{0}}\right)\right]}{\omega-\omega_{0}-i \gamma / 2} d \omega,
\end{aligned}
$$

где $\omega_{0}$ - частота мёссбауэровского перехода в источнике, $\gamma-$ ширина возбужденного уровня, а $c_{0}-$ скорость света в вакууме.

Пусть на пути такого излучения в точке $z=0$ расположен резонансный поглотитель толщиной $d$. Тогда, учитывая явление дисперсии в резонансной среде, поле излучения, прошедшего поглотитель, можно представить в виде $\left[{ }^{2}\right]$ :

$$
\cdot E_{t}(t, d)=\frac{E_{0}}{2 \pi i} \int_{-\infty}^{\infty} d \omega \frac{\exp \left[i \omega\left(t-\frac{d+l}{c_{0}}\right)\right]}{\omega-\omega_{0}-i \gamma / 2} e^{\frac{i b}{\omega-\Omega-i \Gamma / 2}},
$$

где $\Omega$ и $\Gamma$ - частота и ширина резонанса поглотителя,

$$
b=\frac{\Omega_{0}^{2}}{c_{0}} d,
$$

1 ENSV TA Toimetised. $F^{*} M 21987$

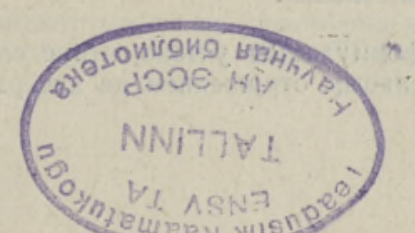




$$
\Omega_{0}^{2}=\frac{4 \pi c_{0}^{3}}{\Omega^{2}} \Gamma_{0} \frac{2 \dot{J}_{+}+1}{2\left(2 J_{0}+1\right)} n_{0} e^{-2 W} .
$$

Здесь $n_{0}-$ концентрация резонансыых ядер в веществе поглотителя, $J$ и $J_{0}-$ спины таких ядер в возбужденном и основном состояниях, $\Gamma_{0}-$ радиационная ширина мёссбауэровского уровня. Фактор ДебаяВаллера $e^{-2 W}$ определяет вероятность бесфононных переходов в веществе поглотителя *.

Проводя в формуле (2) под знаком интеграла Фурье-преобразование, нолучаем

$$
E_{t}(t, d)=E_{i}(t, d)\left[1-b \int_{0}^{t} \frac{J_{1}(2 \sqrt{b \tau})}{\overline{\sqrt{b \tau}}} e^{i \Delta \tau} e^{-\delta \tau} d \tau\right],
$$

где $\Delta-$ изомерный сдвиг $\left(\Delta=\Omega-\omega_{0}\right), \delta=(\Gamma-\gamma) / 2, J_{1}(x)-$ функция Бесселя первого порядка.

Рассмотрим случай толстого поглотителя $(b \gg|\Delta|, b \gg|\delta|)$. Предположим наличие конечного изомерного сдвига $|\Delta| \geqslant(b \gamma)^{1 / 2}$ и малой разницы в ширинах $\Gamma$ и $\gamma\left(|\delta| \ll \Delta^{2} / b\right)$. Оказывается, что в перечисленных условиях в поглотителе возникают два импульса прошедшего излучения, различной длительности и разделенных во времени значительным промежутком $\sim t_{0}=b / \Delta^{2}$. Действительно, при малых временах $t \ll|\Delta|^{-1}$

$$
E_{t}(t, d) \approx E_{i}(t, d) J_{0}(2 \sqrt{b t}),
$$

где $J_{0}(x)$ - функция Бесселя нулевого порядка. Таким образом, при временах $t \ll|\Delta|^{-1}$ поле $E_{t}(t, d)$ имеет вид асимметричного импульса с резким передним фронтом в нулевой момент времени и слабым осциллирующим хвостом. Ширина импульса $\left(\sim b^{-1}\right)$ уменьшается с ростом толщины образца и не зависит от величины изомерного сдвига. Этот импульс является аналогом известного в оптике прекурсного импульса Зоммерфельда $\left[{ }^{4}\right]$.

При больших временах $t \gg|\Delta|^{-1}$ в формуле (5) добавим и вычтем интеграл от $t$ до $\infty$. Тогда поле $E_{t}(t, d)$ принимает вид

$$
E_{t}(t, d)=E_{1}(t, d)+E_{2}(t, d),
$$

где

$$
\begin{gathered}
E_{1}(t, d)=E_{i}(t, d)\left[1-b \int_{0}^{\infty} \frac{J_{1}(2 \sqrt{b \tau})}{\sqrt{b \tau}} e^{i \Delta \tau} e^{-\delta \tau} d \tau\right]= \\
=e^{-\gamma t / 2} e^{-\delta t_{0}} e^{-i \frac{\omega_{0}}{c_{0}} l} E_{p}(t, d), \\
E_{2}(t, d)=E_{i}(t, d) b \int_{t}^{\infty} \frac{J_{1}(2 \sqrt{b \tau})}{\sqrt{b \tau}} e^{i \Delta \tau} e^{-\delta \tau} d \tau .
\end{gathered}
$$

Здесь функция $E_{p}(t, d)$ описывает бегущую волну

$$
E_{p}(t, d)=E_{0} \exp \left[i \omega_{0}\left(t-\frac{d}{c_{0}}+\frac{b}{\Delta \omega_{0}}\right)\right] .
$$

* В формуле (2) учтено, что в случае $\gamma$-излучения $\Omega \gg \Gamma$ и $\Omega \gg \Omega_{9}^{2} / \Gamma$, и пренебрегается слабым отражением на поверхностях поглотителя. 
При определении компонента $E_{2}(t, d)$, связанного с интегралом по $\tau$ от $t$ до $\infty$ (см. $\left.\left(8^{\prime}\right)\right)$, используем известное асимптотическое выражение для функции Бесселя при больших значениях аргумента

$$
\begin{aligned}
& \left(J_{1}(x) \approx(2 / \pi x)^{1 / 2} \cos \left(x-\frac{3}{4} \pi\right)\right) \text {. Тогда } \\
& E_{2}(t, d) \approx E_{i}(t, d) \frac{b}{\sqrt{\pi}} \int_{t}^{\infty} e^{i \Delta \tau} e^{-\delta \tau} \cos \left(2 \sqrt{b \tau}-\frac{3}{4} \pi\right)(b \tau)^{-1 / 4} d \tau .
\end{aligned}
$$

Функция под знаком интеграла в формуле (10) быстро осциллирует везде кроме некоторой области около седловой точки $\tau=t_{0}\left(\left|\tau-t_{0}\right| \leqslant\right.$ $\left.\leqslant 4\left(t_{0} /|\Delta|\right)^{1 / 2}\right)$. Поэтому здесь интегрирование можно провести методом перевала, после чего

$$
\begin{aligned}
E_{2}(t, d) & \approx E_{p}(t, d) \frac{i-1}{2} \sqrt{\frac{2}{\pi}} \int_{y}^{\infty} e^{i x^{2}} d x= \\
& =-E_{p}(t, d) 0,5[1-\Phi(y / \sqrt{i})],
\end{aligned}
$$

где $y=0,5\left(|\Delta| / t_{0}\right)^{1 / 2}\left(t-t_{0}\right)$, а $\Phi(z)$ - интеграл вероятности $(\Phi(z)=$ $\left.=(2 / \pi)^{1 / 2} \int_{0}^{2} e^{-5^{2}} d \xi\right)$. В итоге прошедшее поле при $t \gg|\Delta|^{-1}$ имеет вид импульса

$$
E_{t}(t, d)=e^{-\gamma t / 2} e^{-\delta t_{0}} e^{-i \frac{\omega_{0}}{c_{0}} t} E_{p}(t, d) F\left(\sqrt{\frac{|\Delta|}{t_{0}}}\left(t-t_{0}\right)\right)
$$

где

$$
F(x)=0,5[1+\Phi(x / 2 \sqrt{i})] .
$$

Передний фронт этого импульса двигается со скоростью $v_{g} \approx$ $\approx c_{0}\left(\Delta^{2} / \Omega_{0}^{2}\right)$ по поглотителю и в момент времени $t_{0}$ достигает задней поверхности поглотителя. Скорость $v_{g}$ равна групповой скорости пакета волн со средней частотой $\omega_{0}$, распространяющихся в среде с законом дисперсии $c_{0} k=\omega+\Omega_{0}{ }^{2} /(\Omega-\omega) \quad \vec{k}-$ волновой вектор), причем $v_{g} \ll c_{0} * *$. Форма переднего фронта определяется функцией $F((|\Delta|)$ $\left.\left.\mid t_{0}\right)^{1 / 2}\left(t-t_{0}\right)\right)$; в точке $t=t_{0}$ она равняется 0,5 , а при $\left|t-t_{0}\right| \gg\left(t_{0} /|\Delta|\right)^{1 / 2}$ стремится соответственно к нулю $\left(t-t_{0}<0\right)$ или к единице $\left(t-t_{0}>0\right)$. Длительность импульса определяется множителем $e^{-v t}$, т. е. временем затухания первичного поля излучения.

Описанное выше возникновение двух импульсов $\gamma$-излучения при прохождении резонансной среды значительной толщины имеет следующее физическое объяснение. Поскольку поляризация резонансной среды поглотителя возникает за конѐчное время, то передний фронт падающего поля излучения при прохождении поглотителя не претерпевает изменений. Лишь за время $t \sim b^{-1}$ в поглотителе возникает заметная ядерная поляризация, вследствие чего поле в толщине образца значительно убывает, чему соответствует конечная длительность первого импульса (формула (6)).

Одновременно происходит сильная поляризация вещества в переднем слое поглотителя, достигающая за время $t \sim|\Delta|^{-1}$ равновесное по отношению падающему полю значение. В результате образуется некоторая стационарная бегущая волна поляризации (формула (12)), пе-

\footnotetext{
** При достаточно малых изомерных сдвигах $v_{g}$ может оказаться существенно меньше скорости звука в веществе поглотителя. Так, например, в случае ядра ${ }^{57} \mathrm{Fe}$ частота $\Omega_{0} \sim 10^{-4}-10^{-5} 9 \mathrm{~B}$, и при $|\Delta| \leqslant 5 \Gamma v_{g}<10^{5}$ см $/$ сек.
} 
редний фронт которой двигается в поглотителе со скоростью $v_{g}$ $\sim c_{0}\left(\Delta / \Omega_{0}\right)^{2}$, и в момент $t_{0}=d / v_{\mathrm{g}}$ достигает задней поверхности образца. Отметим, что в оптике такие стационарные состояния системы электромагнитного поля и резонансной среды получили название поляритонных $\left[{ }^{5}\right] * * *$. Поэтому рассмотренную выше волну ядерной поляризации можно назвать ядерным поляритоном.

Подчеркнем, что экспериментальное определение времени $t_{0}$ как функции изомерного сдвига в образцах различной толщины позволяет установить закон дисперсии ядерного поляритона.

Выше рассматривалось прохождение одиночного $\gamma$-импульса через резонансную среду. Можно, однако, показать, что аналогичная перестройка падающего поля происходит и при стационарном потоке $\gamma$-импульсов, если использовать ступенчатую модуляцию резонансного излучения $\left[{ }^{6,7}\right]$. Указанная модуляция выделяет из поля хаотических импульсов сигнал вида (1) [ $\left.{ }^{7}\right]$. В этом случае, однако, второй поляритонный импульс должен существенно укорачиваться вследствие интерференции поля типа (1) и полей хаотических импульсов.

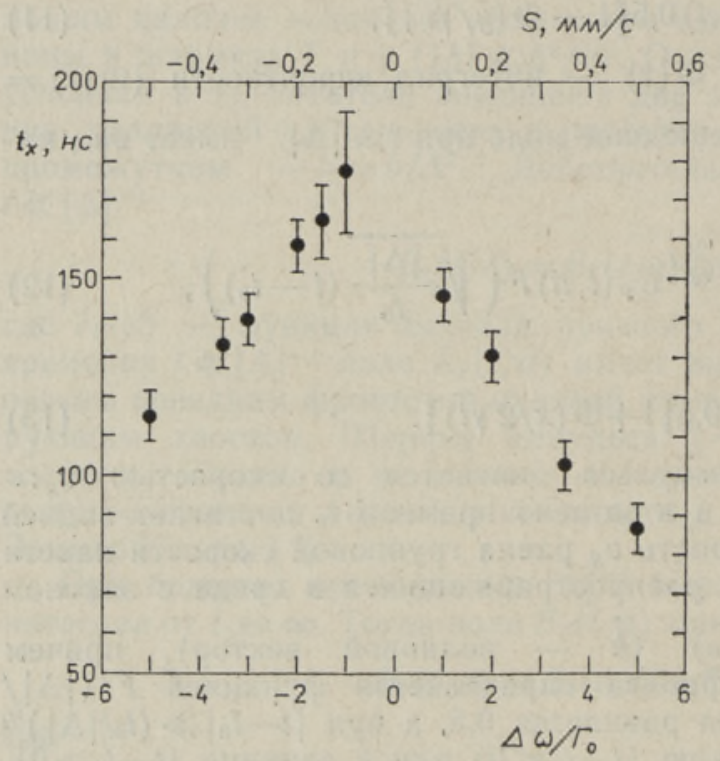

Зависимость момента времени $t_{\mathrm{x}}$, при котором переходный импульс в пропускании ступенчато модулированного мёссбауэровского гамма-излучения ${ }^{57} \mathrm{Fe}$ имеет минимальную интенсивность, от изомерного сдвига s. Источник ${ }^{57} \mathrm{Co}(\mathrm{Pd})$ на пьезопреобразователе из кварца $x$-среза совершает периодические скачкообразные сдвиги длительностью $~ 80$ нс, возбуждаемые прямоугольными импульсами напряжения $u=15$ В, 3 мкс, 123,5 кГц. Поглотителем служит тонкий слой $\mathrm{K}_{4}{ }^{57} \mathrm{Fe}(\mathrm{CN})_{6}$. . $3 \mathrm{H}_{2} \mathrm{O}$ мёссбауэровской толщиной $T_{M}=8 \pm 2$. Различные значения изомерного сдвига $\Delta$ создавались с помощью медленной $(\sim 10$ Гц $)$ допплеровской скорости источника относительно поглотителя. Значения $t_{\mathrm{x}}$ даны относительно фронта возбуждающих фазовую модуляцию излучения импульсов.

Эксперимент описанного выше типа был нами выполнен (см. рисунок). В нем, действительно, наблюдалась четкая зависимость времени $t_{0} \sim t_{x}$ появления прошедшего поглотитель импульса от изомерного сдвига $s \sim \Delta$, которая качественно согласуется с изложенной теорией.

В заключение отметим, что аналогичные эффекты должны возникать и в оптике при прохождении светового импульса вида (1) через резонансный поглотитель, в спектре поглощения которого имеются узкие бесфононные линии.

*** Здесь имеется в виду стационарность по отношению к переходным. процессам. По мере затухания падающего поля излучения поляритонное поле, естественно, также затухает по закону $e^{-\gamma^{t}}$ (формула (12)). 
1. Rebane, K. K. J. Luminescence, 18/19, part 2, 1-9 (1979).

2. Lynch, F. I., Holland, R. E., Hamermesh, M. Phys. Rev., 120, № 2, 513-520 (1960).

3. Harris, S. M. Phys. Rev., 124, № 4, 1178-1185 (1961).

4. Frankel, M. J., Birman, J. L. Phys. Rev., A15, № 5, $2000-2008$ (1977).

5. Агранович В. М., Конобеев Ю. В. Физ. твердого тела, 3, № 2, 360-369 (1961),

6. Helistö, P., Ikonen, E., Katila, T., Riski, K. Phys. Rev. Lett., 49, № 16, 1209-1213 (1982).

7. Реало Э. Х., Хаас М. А., Йги Я. Я. Ж. эксперим. и теорет. физ., 88, № 5, 1864$1872(1984)$.

Институт физики

Академии наук Эстонской ССР
Поступила в редакцию 5/VI 1986

\section{E. REALO, M. HAAS, V. HIZNJAKOV}

\section{TUUMAERGASTUSE KOHERENTNE LIIKUMINE MOSSBAUERI NEELAJAS}

Artiklis on vaadeldud üleminekuprotsesse, mis tekivad Mössbaueri kiirguse impulsi läbiminekul paksust neelajast küllalt suure isomeerse nihke korral. On näidatud, et algne impulss muundub läbiminekul. Seejuures tekivad 1) lühike impulss alghetkedel (optikast tuntud Sommerfeldi prekursse impulsi analoog) ja 2) keskkonnas tuumapolarisatsiooni liikumisega seotud pikk impulss. Vastava lainepaketi grupikiirus on oluliselt väiksem valguse kiirusest vaakumis ning mõlema impulsi vahelise ajalise viivise kaudu eksperimentaalselt määratav. On toodud katsetulemused, mis kvalitatiivselt on koøskõlas arendatud teooriaga.

\section{E. REALO, M. HAAS, V. HIZHNYAKOV}

\section{COHERENT MOTION OF NUCLEAR EXCITATION IN A MOSSBAUER ABSORBER}

A transient-pulse of a Mössbauer radiation in a thick absorber on a significant isomer shift is considered. It is shown that in transmission the primary pulse decomposes into 1) a short pulse at initial time moments (an analog of the Sommerfeld precursive pulse known in optics) and 2) a prolonged pulse connected with the motion of the wave of nuclear polarization in the medium. The group velocity of such a wave packet is considerably lower than the velocity of light in the vacuum and it can be determined experimentally by the time delay between the two pulses. The results of an experiment employing stepwise modulation are in a qualitative agreement with the developed theory. 